\title{
Novel insulin products: Why would patients, professionals and industry want them?
}

\author{
VICKY STOKES, ${ }^{1}$ KAVITHA SEBASTIAN ROZARIO, ${ }^{2}$ JYOTHIS GEORGE ${ }^{3}$
}

\begin{abstract}
Multiple innovations over a century have improved the safety, tolerability and clinical acceptability of insulin. From a therapeutic option that required multiple injections a day, often with a poor predictability, insulin has evolved into a treatment approach that an empowered patient can self-administer safely. It is the most potent glucose-lowering therapy and has hence seen widespread clinical adoption in the last two decades in the treatment of type 2 diabetes.

At the same time, the unit-costs of insulin have not declined as is often the case with long established therapies. We review the history of insulin as a drug, charting its course from early experiments to modern insulin therapies. We consider the need (or lack thereof) for novel insulin from the perspectives of patients, healthcare professionals and healthcare payers.

Whilst there are many valid arguments to support the development of novel insulins, healthcare systems and payers will require a clear demonstration of value for any novel insulin product. If a premium price (i.e. a higher price than comparable products) is demanded by manufacturers, healthcare payers would rightly seek evidence of added value - this could involve clear markers of increased safety and efficacy and/or the offsetting of other diabetes-related costs.

Br J Diabetes 2016;16:198-201
\end{abstract}

Key words: insulin, biosimilar, glycaemic control

Insulin as therapy for diabetes: a gift to mankind

One hundred years ago (in 1916) Nicolae Paulescue, a Romanian Professor of Physiology, developed a pancreatic extract that had a normalising effect on blood glucose levels in a dog with diabetes. Unfortunately, World War I interrupted his experiments and he did not publish until much later. Almost a decade earlier Georg Ludwig

Oxford Centre for Diabetes, Endocrinology and Metabolism, Churchill Hospital, Oxford, UK

Paediatric Endocrinology, John Radcliffe Hospital, Oxford, UK.

Boehringer Ingelheim Ltd, Bracknell, UK; Warwick Medical School,

University of Warwick, Coventry, UK; Diabetes and Endocrinology, Royal

Berkshire Hospital, Reading, UK

Address for correspondence: Dr Vicky Stokes

Oxford Centre for Diabetes, Endocrinology and Metabolism, Churchill

Hospital, Oxford, UK

Tel: $+44(0) 1865857340$

E-mail: vickystokes22@hotmail.com

http://dx.doi.org/10.15277/bjd.2016.110
Zülzer, a physician from Berlin, published research into treating diabetes with pancreatic extracts but, again, the Great War interrupted research efforts. The latter efforts were in partnership with a German company called Farbwerke Hoechst, whose successors developed another popular insulin almost a century later - insulin HOE901, now known as glargine.

Halfway across the world, Canadian orthopaedic surgeon, Frederick Banting, and his medical student assistant, Charles Best, successfully isolated insulin in 1921. Famously, this extract was first tested on "Marjorie", a dog with surgically induced diabetes, and they managed to keep her alive all summer. After this initial success, the head of the laboratory, Professor MacLeod, and his team helped to refine the production (for example, using cows rather than dogs) and the purification of insulin. Just 20 weeks later the extract was ready to be given to human patients. The first was Leonard Thompson, a 14-year-old who lay dying from type 1 diabetes in Toronto General Hospital. He suffered an allergic reaction to his first injection which led to frantic revisions over the next 2 weeks. His second injection produced dramatic results and Leonard lived another 13 years before dying of pneumonia.

The University of Toronto did attempt to produce insulin for use by patients, but it soon realised that it lacked the expertise to produce it on a commercial scale. Intense research and a collaboration with Eli Lilly followed to allow rapid distribution of insulin worldwide. From May to November 1922, output trebled every month and, by early 1923 - about one year after the first test injection insulin became widely available. The incidence of death by diabetic coma dropped from 63.8\% (1898-1914) to 8.3\% (1922-36), and then again to just 1.3\% (1950-1957).

Banting and MacLeod were awarded the Nobel Prize for their discovery in 1923. Banting chose to share his prize with his medical student, Best. This is not the only noble gesture that the team is famous for - Banting and Best sold their insulin patent to the University of Toronto for a dollar, noting that "When the details of the method of preparation are published, anyone would be free to prepare the extract but no one could secure a profitable monopoly". Whilst exclusive agreements were drawn with Lilly for commercialisation of insulin in the USA, licences were offered to many other companies in various countries. Among these licensees was Nordisk Insulin Laboratorium - which subsequently merged with another Danish company (Novo Terapeutisk Laboratorium) to form Novo Nordisk. ${ }^{1}$

Why isn't insulin 'generic' yet?

Given almost a century of research and development that underpin modern insulin therapy, most patients (at least in the developed 
world) continue to be treated with insulins that are not generic. This is different from well-established therapies such as aspirin or metformin. Why is this? This is a question that exercises many minds, ${ }^{1}$ especially those concerned about the high costs of insulins. To answer this important question we need to look at the series of innovations since the original discovery of insulin.

\section{Early innovations}

Banting's early preparation of insulin was a short-acting formulation, necessitating multiple injections each day. In 1936, Hans Christian Hagedorn discovered that the action of insulin can be prolonged by the addition of protamine obtained from salmon semen (milt). Commercial and regulatory pressures meant it took 14 years for this discovery to be commercialised as NPH, an intermediate-acting insulin (Neutral Protamine Hagedorn). Unlike Banting and Best (whose patent applications were filed only after the first patient was treated), intellectual property underpinning NPH was secured four years before its launch in 1950.

\section{Human insulin}

Soon after NPH hit the markets, another key discovery was made. In 1955, Frederick Sanger made insulin the first protein to be fully DNA sequenced and was awarded the Nobel Prize in 1959. Following on from this work, the first synthetic 'human' insulin was produced in the early 1960s by Panayotis Katsoyannis, University of Pittsburgh, and Helmut Zahn, RWTH Aachen University, simultaneously. In 1978, insulin became the first human protein to be manufactured through biotechnology using $\mathrm{E}$. coli as part of a collaboration between Genentech and the Beckman Research Institute. This group formed a larger collaboration with Eli Lilly and went on to produce the first available synthetic insulins approved by the FDA in 1982. Two preparations were marketed - Humulin R (regular) and Humulin N (NPH).

The discovery and commercialisation of human insulin fundamentally changed the dynamics of insulin supply. Until then, all available insulin preparations were purified from animal sources. Production of insulin required a regular supply of pancreata from slaughtered animals. Insulin manufacturers all had contracts with abattoirs and, in some cases, the pancreata were brought from many abattoirs in refrigerated vans to the production facility to be stored in refrigerated cellars. This had raised questions about whether manufacturing can be scaled up to meet rising demand, but also whether the quality of these insulins could be consistent. Production of recombinant insulin, however, is a scalable process (albeit requiring a large amount of capital investment) and end-toend quality control systems can be reliably implemented.

\section{Analogue insulin}

Once the commercial synthesis of human sequence insulin was made possible through recombinant technology, it was merely a question of time before this sequence was systematically modified to alter insulin pharmacokinetics. This technology took synthetic insulin and genetically modified the amino acid sequence to change its profile of action, i.e. for short-acting insulins to act more quickly and long-acting insulins to have a flatter profile.
First on to the market (in the 1990s) were single amino acid substitutions that made insulin 'faster acting'. This provided patients and professionals with a tool that had at least a theoretical likelihood of achieving meal-time coverage that was more physiological. Insulin Lispro was approved in 1996, with the two other leading players - aspart and glulisine - following in 2000 and 2004, respectively. With the advent of these fast-acting insulins, which temporally coincided with the publication of DCCT results, treatment paradigms also began to shift - with 'basal-bolus' regimes coming into vogue, aiming to achieve tighter and 'more physiological' glycaemic profile.

At the turn of the millennium a long-acting analogue of insulin (glargine) was launched. As NPH provides less than 24-hour basal cover with a variable peak, a longer-acting analogue with a 'flat' pharmacokinetic profile was hypothesised to improve glycaemic control with lower frequency of attendant hypoglycaemia. Glargine was launched with an easy-to-titrate algorithm, and trained primary care professionals to use insulin. This package of improvements was accepted widely by the diabetes community, leading to glargine becoming the first insulin to achieve annual sales in excess of 5 billion US dollars. ${ }^{2}$

In summary, whilst 'insulin' has been around for the best part of a century, it has had the benefit of a number of innovations (including Nobel Prize winning ones). These have led to improvements in pharmacokinetics and tolerability of insulin therapy. However, they have also required vast injections of capital and, under our current international norm to reward innovation, novel products were being given patent protection. Although much of this intellectual property protection has since expired, ${ }^{3}$ the relatively high costs of synthesising and marketing a large biological molecule (more on this below) all create a high cost of entry for new competitors to the insulin market.

\section{Do patients need novel insulins?}

\section{The case for}

Despite a century of research and development, insulin therapy remains a challenging prospect for many patients. Specific challenges include:

1. Optimal insulin therapy (at least in patients with type 1 diabetes) requires multiple injections in a day - whilst these injections are less painful than they were, the discomfort hasn't been completely eradicated.

2. Few if any other medications require as close monitoring of their respective pharmacological effect. Patients need to test their blood glucose levels (again, multiple times a day in many cases) to ensure they are dosing themselves correctly.

3. There are no other drugs where patients need to undertake complex mathematical calculations multiple times a day to accurately dose themselves. The costs of getting these wrong range from suboptimal control, to modest hypos, to catastrophic incapacitation.

In addition, there are practical challenges such as maintaining insulin cold chain and the hassle of carrying all the injection paraphernalia. Little surprise that, on a global level, suboptimal 
glycaemic control remains the norm - rather than the exception it ought to be. It could therefore be strongly argued that continuing to improve the formulations and delivery mechanisms of insulin products is desirable from the patients' perspective.

\section{The case against}

It could be argued that what patients need is more education and support from healthcare professionals and others, allowing better use of existing insulins more effectively rather than newer insulins. Comparisons have been drawn with sport - i.e. providing an amateur golfer with the most expensive set of clubs will not make him a champion golfer. ${ }^{4}$

However, an equally strong case could be made for making insulin therapy a lot easier for patients to self-administer and selftitrate. Sticking to the sporting analogy, the aim is not to have tools that benefit the elite professionals but something that amateurs can easily master too. A fair comparison can be drawn from how oral anticoagulation therapy has moved on from warfarin (requiring frequent monitoring, but nevertheless leading to chronic suboptimal anticoagulation) to simpler novel oral agents. Given that the majority of healthcare costs in modern healthcare systems are driven by salaries for professionals, the emphasis needs to be on making patients as self-reliant as they can be.

\section{Why do insulin manufacturers need novel insulins?}

Drug classes led by generic drugs (e.g. antibiotics) do not attract much in way of research investment - merely because there is no reliable mechanism for that investment to be paid back. By continually developing novel products (and successfully commercialising them with protection of intellectual property), the industry is able to invest in further innovation. Clearly, not all the profits are re-invested into R\&D; some go towards rewarding investors and shareholders. Whilst some may find it objectionable for individuals or corporations to profit from medicines such as insulin, the fact remains that we have no other models for rewarding risky investments into developing medicines. Some have therefore pointedly observed that it is only the parts of the world with the capitalist system that have given medicine phenomenal therapeutic progress across all specialties. ${ }^{4}$

As newer insulins were licensed and brought to market, older versions of insulins went out of production and supply - although many would argue that they were still 'usable' in a number of patients. ${ }^{4}$ In this regard, the insulin market has behaved much more like consumer technology markets to avoid 'commoditisation' of its product. Readers will note that manufacturers of computers or mobile phones actively remove older models from the market as newer models are launched. Another reason to remove older products is merely one of capacity - manufacturing and distributing a biological product like insulin is capital intensive. When one adds the ongoing costs of marketing (and mandatory activities related to marketing an approved product such as pharmacovigilance), it is uneconomical for any commercial organisation to market multiple drugs that address the same medical need. In this context, companies have - perhaps understandably - selected insulin products with the strongest intellectual property protection and therefore the strongest competitive differentiation.
In summary, the cyclical launches of novel insulin products provide companies with a mechanism to generate sufficient profits to invest them (at least partly) into further research.

\section{Do healthcare systems and payers need novel insulins?}

\section{The case for}

With one in 11 adults currently estimated to have diabetes, ${ }^{5}$ its treatment is surely at the forefront of healthcare managers worldwide. With further increases predicted in diabetes prevalence over the next few decades, ever more 'systemic' attention could (and should) fall on diabetes.

At a health system level, diabetes is an expensive condition to treat. In the UK around 10\% of the NHS budget (approximately $\mathrm{f} 10$ billion) is spent on diabetes. However, the lion's share of this is spent treating the complications of poorly controlled diabetes such as renal failure, retinopathy, amputations and cardiovascular disease. ${ }^{6}$ As at least half of all patients remain at suboptimal control, it is clearly in the long-term interest of health economies to invest in treatments that prevent such complications. Controlling blood glucose certainly appears to play a part in preventing these complications, and almost all patients with diabetes will require insulin if they survive for long enough after its diagnosis.

\section{The case against}

Total annual sales of insulin increased from about \$2 billion in 1995 to about $\$ 20$ billion in 2014. Much of this increase is explained by the increasing number of patients being treated with insulin - due to increasing prevalence of diabetes as well as changes in physician attitudes triggered by the DCCT and UKPDS studies. In other markets, increasing demand for a particular technology leads to increasing competition, thus triggering downward pressure on prices. This trend has been largely absent in the insulin space, as we have already noted.

Irrespective of the healthcare delivery mechanism, increasing healthcare costs have an impact on public finances. In publicly funded healthcare models (such as the NHS in the UK), these costs are directly observable. Even in heterogeneous healthcare systems (e.g. USA), it could be argued that increasing expenditure on insulin diverts capital and other resources away from other (arguably more needy) areas of the healthcare economy.

Given the rapid increase in the number of patients requiring insulin therapy, healthcare systems are therefore understandably focused on saving costs. Somewhat disappointingly, it is often only the direct acquisition costs (i.e. price paid for insulin per se) that is considered. Putative savings on ancillary costs (e.g. glucose monitoring) and on complications (e.g. hypoglycaemia), which could impact adherence, are often not fully appreciated.

In summary, healthcare systems and payers will require a clear demonstration of value for any novel insulin product. In other words, if a premium (i.e. a higher price than comparable products) is demanded by manufacturers, payers would rightly seek evidence of added value (which may involve clear markers of increased safety and efficacy and/or the offsetting of other diabetes-related costs). 


\section{What are biosimilar insulins and how will they influence diabetes management?}

Biosimilar insulins are identical in their amino acid sequence to an existing insulin product, technically called 'reference insulin'. Whilst they sound similar to how 'generic' versions of off-patent small molecule drugs (e.g. statins) are brought to market, the development of 'biologics' like insulin presents a number of challenges, both in terms of production and regulatory review. Insulin is a large and complex protein manufactured by biotechnology, so the exact reproduction of existing medications is challenging. Small differences in the manufacturing process can potentially impact on the final product, so new products will need to meet the criteria set by regulators, such as the EMA and FDA, to be licensed. Furthermore, unlike small molecules where the introduction of generic products leads to a marked drop in prices, biosimilar products are often priced at less of a discount than existing products - reflecting their higher development and manufacturing costs.

Creating a biosimilar for insulin glargine seems to be attracting the most commercial interest. Globally, there are several marketed preparations available that have not been subjected to internationally accepted trial evaluation and regulatory approval from regulatory agencies such as the FDA or EMA. For example, Basalin has been marketed in China since 2005 by Gan \& Lee Pharmaceuticals, 7 but a dossier submitted by a local company (LaFranCol, Cali) was rejected in 2009 in Columbia due to lack of immunogenicity data. ${ }^{8}$ Bonglixan is marketed in Mexico but has no widely available trial data behind it. ${ }^{9}$ Biocon, an Indian manufacturer, also has a biosimilar glargine on the market in India. ${ }^{10}$

Lilly and Boehringer Ingelheim have brought to market the first biosimilar glargine approved by the FDA and EMA (marketed as Abasaglar in the UK). Abasaglar became available in the UK in September 2015. It has been shown to be as effective as glargine in lowering $\mathrm{HbA}_{1 \mathrm{c}}$ in patients with type 1 and type 2 diabetes, with a comparable side-effect profile. Cartridges are 100 units $/ \mathrm{ml}, 5 \times 3 \mathrm{ml}$ for the pen or Abasaglar pre filled pen 100 units $/ \mathrm{ml}, 5 \times 3 \mathrm{ml}$ is f35.28 (excluding VAT). ${ }^{11}$ This is $15 \%$ less than the cost of Lantus, where $5 \times 3 \mathrm{ml}$ Solostar prefilled pens cost $\mathrm{f} 40.36 .{ }^{12}$

\section{Combining GLP-1 compounds with insulin: why and how?}

Insulin was initially developed for the treatment of type 1 diabetes but, over the years, the vast majority of patients who use insulin to control their blood glucose concentrations have been patients with type 2 diabetes. This move was augmented with the arrival of longacting insulin analogues.

Large proportions of patients with diabetes require multiple glucose-lowering medications to achieve glycaemic control. Fixed-dose combinations of multiple diabetes drugs within one formulation provide the opportunity to reduce 'pill count' for patients, potentially improving adherence. It has become common practice to combine metformin with other agents but, more recently, the FDA has also approved other combinations (e.g. empagliflozin + linagliptin). Combinations of insulin with other injectable diabetes therapies are considered to have the same advantages. For example, GLP-1 in fixed combination with insulin initiated in a slow stepwise manner

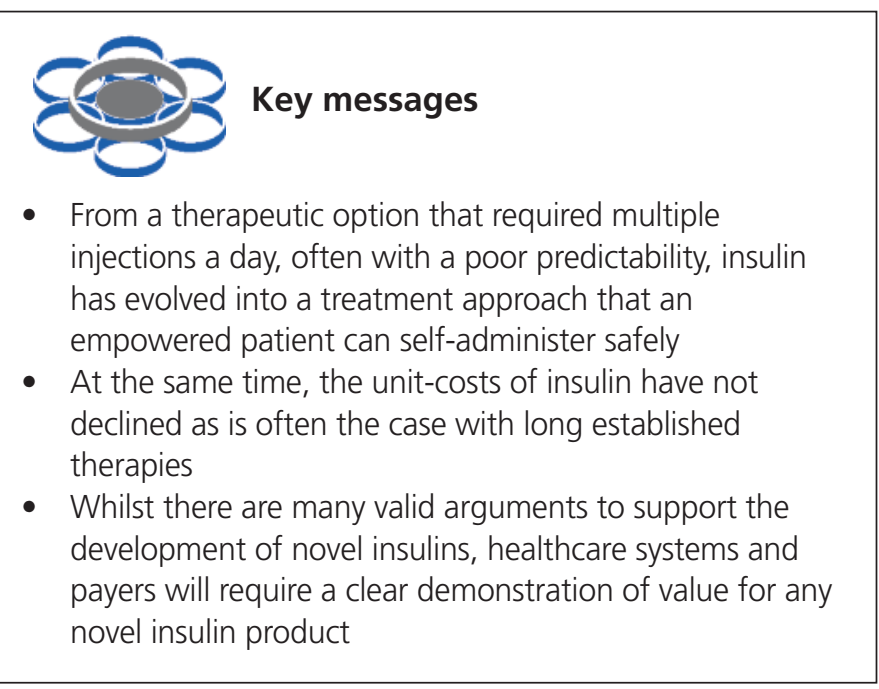

appears to be associated with fewer gastrointestinal side-effects potentially further enhancing compliance.

\section{Conclusions}

The future of the insulin and injectable therapies market continues to evolve. The situation we see now is very different from the historical picture. Given the wide range of insulin types and therapeutic interventions, we are in a potentially much better position when it comes to selecting the right combination for our patients. It's clear that there is vast public, scientific and commercial interest in meeting the demand for insulin research and innovation.

Conflict of interest VS and KSR have no potential conflicts to disclose JG is a Global Senior Medical Director for Boehringer Ingelheim who, in an alliance with Eli Lilly, markets the biosimilar insulin Abasaglar in the UK.

Funding None.

\section{References}

1. Greene JA, Riggs KR. Why is there no generic insulin? Historical origins of a modern problem. N Engl J Med 2015;372:1171-5. http://dx.doi.org/10.1056/NEJMms1411398

2. PM Live website. http://www.pmlive.com/top_pharma_list/pharmaceutical_products/lantus

3. Conterno E. For some diabetics, a struggle to pay for insulin. New York Times, 29 Feb 2016

4. Gough S, Russell-Jones D, Millar P, Sumner J. Re: Big changes led to big profits for insulin manufacturers (rapid response). BMJ 21 Jan 2011. http://www.bmj.com/content/341/bmj.c7139/rapid-responses

5. International Diabetes Federation. IDF Diabetes Atlas. 7th Edition http://www.diabetesatlas.org/

6. Diabetes UK. The Cost of Diabetes Report. 2014. https://www diabetes.org.uk/Documents/Diabetes\%20UK\%20Cost\%20of\%20Diabetes\%20Report.pdf

7. Gan \& Lee Pharmaceuticals website http://www.ganlee.com/en/index.php?page $=2$

8. Owens DR, Landgraf W, Schmidt A, Bretzel RG, Kuhlmann MK. The emergence of biosimilar insulin preparations - a cause for concern? Diabetes Technol Ther 2012;14:989-96. http://dx.doi.org/10.1089/ dia.2012.0105

9. Gough S. Biosimilar insulins: opportunities and challenges. Practical Diabetes 2013:30:146-7. http://dx.doi.org/10.1002/pdi.1763

10. Biocon Pharmaceuticals website.

http://www.biocon.com/biocon_products_bio_BF_basalog_mp.asp

11. Basalin (2015). MIMS. Retrieved from www.mims.com

12. Lantus (2015). BNF 61. 